\title{
Linear Transformations between Multipartite Quantum Systems That Map the Set of Tensor Product of Idempotent Matrices into Idempotent Matrix Set
}

\author{
Jinli Xu, ${ }^{1}$ Baodong Zheng, ${ }^{1}$ and Hongmei Yao $^{2}$ \\ ${ }^{1}$ Department of Mathematics, Harbin Institute of Technology, Harbin 150001, China \\ ${ }^{2}$ College of Science, Harbin Engineering University, Harbin 150001, China \\ Correspondence should be addressed to Baodong Zheng; zbd@hit.edu.cn
}

Received 12 May 2013; Accepted 16 July 2013

Academic Editor: Gen-Qi Xu

Copyright (C) 2013 Jinli Xu et al. This is an open access article distributed under the Creative Commons Attribution License, which permits unrestricted use, distribution, and reproduction in any medium, provided the original work is properly cited.

Let $H_{n}$ be the set of $n \times n$ complex Hermitian matrices and $\mathscr{P}_{n}$ (resp., $\mathscr{T}_{n}$ ) be the set of all idempotent (resp., tripotent) matrices in $H_{n}$. In $l$-partite quantum system $H_{m_{1} \cdots m_{l}}=\otimes_{1}^{l} H_{m_{i}}, \otimes_{1}^{l} \mathscr{P}_{m_{i}}\left(\right.$ resp., $\left.\otimes_{1}^{l} \mathscr{T}_{m_{i}}\right)$ denotes the set of all decomposable elements $\otimes_{1}^{l} A_{i}$ such that $A_{i} \in \mathscr{P}_{m_{i}}$ (resp., $\left.A_{i} \in \mathscr{T}_{m_{i}}\right)$. In this paper, linear maps $\phi$ from $H_{m_{1} \cdots m_{l}}$ to $H_{n}$ with $n \leq m_{1} \cdots m_{l}$ such that $\phi\left(\otimes_{1}^{l} \mathscr{P}_{m_{i}}\right) \in \mathscr{P}_{n}$ are characterized. As its application, the structure of linear maps $\phi$ from $H_{m_{1} \cdots m_{l}}$ to $H_{n}$ with $n \leq m_{1} \cdots m_{l}$ such that $\phi\left(\otimes_{1}^{l} \mathscr{T}_{m_{i}}\right) \in \mathscr{T}_{n}$ is also obtained.

\section{Introduction}

Let $M_{n}$ be the vector space of $n \times n$ complex matrices and $H_{n} \subseteq M_{n}$ be the vector space of $n \times n$ complex Hermitian matrices. In quantum information theory, a $l$-partite system can be represented as the tensor product space $H_{m_{1} \cdots m_{l}} \equiv$ $\otimes_{1}^{l} H_{m_{i}}$, where $\otimes$ is the usual Kronecker product of matrices; a quantum state is represented as a positive semidefinite with trace one in $H_{n}$, see [1]. In quantum information science and quantum computing, it is important to understand, characterize, and construct different classes of maps on quantum states [2]. For example, entanglement is one of the main concepts in quantum information theory, and entangled state involves at least bipartite system or multipartite system [3]; to study entangled states, one should construct entanglement witnesses, which are special types of positive maps, see [4]. On this background, the research on the characterizations of maps leaving invariant, some important subsets or quantum properties attracted more and more researchers' attention, see $([1,3,5])$. Especially, in [5], Lim characterized the linear and additive maps on tensor products of spaces of Hermitian matrices that carry the set of tensor product of rank one matrices into itself.
Preserver problem is a hot area in Banach algebra; there are many results about this area, see ([6-11]). Specially, the idempotent preservers and the rank one preservers play an important role; therefore, it is meaningful to study the two preservers. Chan et al. [12] first characterized linear transformations on $M_{n}$ preserving idempotent matrices. In [13], the authors obtained the following result: linear map $T: H_{n} \rightarrow H_{m}$ with $n \leq m$ satisfies $T\left(\mathscr{P}_{n}\right) \subset \mathscr{P}_{m}$ if and only if $T=0$ or

$$
T(X)=Q X Q^{-1} \quad \text { or } \quad T(X)=Q X^{T} Q^{-1}, \quad \forall X \in H_{n},
$$

where $Q$ is unitary matrix.

Since the study of linear transformations, preserving idempotents is important in many aspects of mathematics and physics, see [14-17], and inspired by the above, the purpose of this paper is to study linear maps from $l$-partite system to the space of Hermitian matrices that carry the set of tensor product of idempotent matrices into the set of idempotent matrices in the space of Hermitian matrices, that is, $\phi: H_{m_{1} \cdots m_{l}} \rightarrow H_{n}$ with $n \leq m_{1} \cdots m_{l}$ satisfying

$$
\phi\left(\otimes_{1}^{l} \mathscr{P}_{m_{i}}\right) \subset \mathscr{P}_{n},
$$


where $\mathscr{P}_{n}$ is the set of all idempotent matrices in $H_{n}$, that is, $\mathscr{P}_{n}=\left\{A \in H_{n} \mid A^{2}=A\right\}$ and $\otimes_{1}^{l} \mathscr{P}_{m_{i}}$ denotes the set of all decomposable elements $\otimes_{1}^{l} A_{i}$ such that $A_{i} \in \mathscr{P}_{m_{i}}$. As application, the forms of linear maps from $l$-partite system to the space of Hermitian matrices that carry the set of tensor product of tripotent matrices into the set of tripotent matrices in the space of Hermitian matrices are obtained, that is, $\phi$ : $H_{m_{1} \cdots m_{l}} \rightarrow H_{n}$ with $n \leq m_{1} \cdots m_{l}$ satisfying

$$
\phi\left(\otimes_{1}^{l} \mathscr{T}_{m_{i}}\right) \subset \mathscr{T}_{n}
$$

where $\mathscr{T}_{n}$ is the set of all tripotent matrices in $H_{n}$, that is, $\mathscr{T}_{n}=\left\{A \in H_{n} \mid A^{3}=A\right\}$ and $\otimes_{1}^{l} \mathscr{T}_{m_{i}}$ denotes the set of all decomposable elements $\otimes_{1}^{l} A_{i}$ such that $A_{i} \in \mathscr{T}_{m_{i}}$.

Throughout this paper, we always assume integers $l \geq 1$ and $n, m_{1}, \ldots, m_{l} \geq 2$. Let $I_{k}$ be the $k \times k$ identity matrix, 0 be the zero matrix which order is omitted in different matrices just for simplicity, and $X^{T}$ (resp., $X^{*}$, rank $X$ ) be the transpose (resp., conjugate transpose, rank) of $X . E_{i j}^{(n)}(1 \leq i, j \leq n)$ stands for the $n \times n$ matrix with 1 at the $(i, j)$ th entry and 0 otherwise. $D_{i j}^{(n)}=E_{i j}^{(n)}+E_{j i}^{(n)}$ and $K_{i j}^{(n)}=\sqrt{-1} E_{i j}^{(n)}-\sqrt{-1} E_{j i}^{(n)}$, where $1 \leq i \neq j \leq n$. For real numbers $a$ and $b$ with $a \leq b$, let $[a, b]$ be the set of all integers between $a$ and $b . \oplus$ is the usual direct sum of matrices. A linear map $\pi=\otimes_{1}^{l} \pi_{i}: H_{m_{1} \cdots m_{l}} \rightarrow$ $H_{m_{1} \cdots m_{l}}$ is canonical if $\pi$ satisfies $\pi\left(\otimes_{1}^{l} A_{i}\right)=\otimes_{1}^{l} \pi_{i}\left(A_{i}\right)$, where $\pi_{i}: H_{m_{i}} \rightarrow H_{m_{i}}$ satisfies $\pi_{i}\left(A_{i}\right)=A_{i}$ or $\pi_{i}\left(A_{i}\right)=A_{i}^{T}$ for all $A_{i} \in H_{m_{i}}$.

\section{Main Results}

Lemma 1 (see [18]). Suppose $P_{1}, \ldots, P_{k} \in \mathscr{P}_{n}$ such that $P_{i}+$ $P_{j} \in \mathscr{P}_{n}, \forall 1 \leq i<j \leq k$. Let $r_{i}=\operatorname{rank} P_{i}$. Then, there exists $a$ unitary matrix $U \in M_{n}$ such that

$$
P_{i}=U \operatorname{diag}(0, \ldots, 0,1, \ldots, 1,0, \ldots, 0) U^{*}
$$

where $\operatorname{diag}(0, \ldots, 0,1, \ldots, 1,0, \ldots, 0)$ is the diagonal matrix in which all diagonal entries are zero except those in the $\left(r_{1}+\cdots+\right.$ $\left.r_{i-1}+1\right)$ st to the $\left(r_{1}+\cdots+r_{i}\right)$ th rows.

Lemma 2. Let $P \in \mathscr{P}_{r}$. Suppose $X, 0_{s} \oplus P \oplus 0_{n-r-s}-X \in \mathscr{P}_{n}$. Then, there exists $X_{r} \in \mathscr{P}_{r}$ such that $X=0_{s} \oplus X_{r} \oplus 0_{n-r-s}$, where $0 \leq r, s \leq n$.

Proof. Let $T \in M_{r}$ be a unitary matrix satisfying $P=T\left(I_{t} \oplus\right.$ $0) T^{*}$. Set

$$
\left[\begin{array}{ccc}
I_{r} & 0 & 0 \\
0 & T^{*} & 0 \\
0 & 0 & I_{n-r-s}
\end{array}\right] X\left[\begin{array}{ccc}
I_{r} & 0 & 0 \\
0 & T & 0 \\
0 & 0 & I_{n-r-s}
\end{array}\right]=\left[\begin{array}{ccc}
X_{11} & X_{12} & X_{13} \\
X_{21} & X_{22} & X_{23} \\
X_{31} & X_{32} & X_{33}
\end{array}\right],
$$

where $X_{11} \in H_{s}, X_{22} \in H_{t}$. Then,

$$
\begin{gathered}
{\left[\begin{array}{lll}
X_{11} & X_{12} & X_{13} \\
X_{21} & X_{22} & X_{23} \\
X_{31} & X_{32} & X_{33}
\end{array}\right] \in \mathscr{P}_{n},} \\
{\left[\begin{array}{ccc}
0_{r} & 0 & 0 \\
0 & I_{t} & 0 \\
0 & 0 & 0
\end{array}\right]-\left[\begin{array}{lll}
X_{11} & X_{12} & X_{13} \\
X_{21} & X_{22} & X_{23} \\
X_{31} & X_{32} & X_{33}
\end{array}\right] \in \mathscr{P}_{n} .}
\end{gathered}
$$

A straightforward computation shows that $X=0_{s} \oplus\left[T\left(X_{22} \oplus\right.\right.$ $\left.\left.0_{r-t}\right) T^{*}\right] \oplus 0_{n-r-s}$; therefore, the result holds.

Lemma 3. Let $A=P_{r} \oplus 0_{s} \oplus 0_{n-r-s}$ and $B=0_{r} \oplus P_{s} \oplus 0_{n-r-s}$ with $P_{r} \in \mathscr{P}_{r}, P_{s} \in \mathscr{P}_{s}$. If

$$
\alpha A+\beta B+\gamma X \in \mathscr{P}_{n} \quad \text { for any real } \gamma \text { with } 0 \neq|\gamma| \leq \frac{1}{2},
$$

where $X \in M_{n}, \alpha$ and $\beta$ are all roots of $x^{2}-x+\gamma^{2}=0$, then, $X^{2}=A B$, and $X$ is of the form

$$
\left[\begin{array}{cc}
0_{r} & X_{12} \\
X_{21} & 0_{s}
\end{array}\right] \oplus 0_{n-r-s}
$$

where $X_{12} \in M_{r \times s}, X_{21} \in M_{s \times r}$.

Proof. It is clear that $A, B \in \mathscr{P}_{n}$ and $A B=B A=0$. By a direct computation, one can obtain that

$$
X=\alpha(A X+X A)+\beta(B X+X B)+\gamma\left(X^{2}-A-B\right) .
$$

Replacing $\gamma$ by $-\gamma$, we have $X^{2}=A+B$ and

$$
X=\alpha(A X+X A)+\beta(B X+X B) .
$$

Choosing $\gamma=2 / 5$, then $\alpha=1 / 5, \beta=4 / 5$ or $\alpha=4 / 5, \beta=1 / 5$, this yields that

$$
\begin{aligned}
& X=\frac{1}{5}(A X+X A)+\frac{4}{5}(B X+X B), \\
& X=\frac{4}{5}(A X+X A)+\frac{1}{5}(B X+X B) .
\end{aligned}
$$

Thus, $X=A X+X A$ and $X=B X+X B$. This, together with the form of $A$ and $B$, implies that $X$ has the form $\left[\begin{array}{cc}0_{r} & X_{12} \\ X_{21} & 0_{s}\end{array}\right] \oplus 0_{n-r-s}$, where $X_{12} \in M_{r \times s}, X_{21} \in M_{s \times r}$.

Lemma 4. Let $J=P_{p} \oplus-P_{q} \oplus 0_{n-p-q}$ with $P_{p} \in \mathscr{P}_{p}$ and $P_{q} \in$ $\mathscr{P}_{q}$. Suppose $X \in \mathscr{T}_{n}$ satisfying $J-X \in \mathscr{T}_{n}$ and $J-2 X \in \mathscr{T}_{n}$. Then, $X=X_{p} \oplus-X_{q} \oplus 0_{n-p-q}$ with $X_{p} \in \mathscr{P}_{p}$ and $X_{q} \in \mathscr{P}_{q}$.

Proof. By a direct computation, we have

$$
\begin{aligned}
& 3 X=J^{2} X+J X J+X J^{2}, \\
& 3 X=J X^{2}+X J X+X^{2} J .
\end{aligned}
$$


Let $U \in M_{p}$ and $V \in M_{q}$ be unitary matrices satisfying $P_{p}=$ $U\left(I_{r} \oplus 0_{p-r}\right) U^{*}$ and $P_{q}=V\left(I_{s} \oplus 0_{q-s}\right) V^{*}$, respectively. Then,

$$
\begin{gathered}
{\left[\begin{array}{ccc}
U^{*} & 0 & 0 \\
0 & V^{*} & 0 \\
0 & 0 & I_{n-p-q}
\end{array}\right] J\left[\begin{array}{ccc}
U & 0 & 0 \\
0 & V & 0 \\
0 & 0 & I_{n-p-q}
\end{array}\right]} \\
=\left[\begin{array}{ccc}
I_{r} \oplus 0_{p-r} & 0 & 0 \\
0 & -I_{s} \oplus 0_{q-s} & 0 \\
0 & 0 & 0_{n-p-q}
\end{array}\right] .
\end{gathered}
$$

By (12), one can assume that

$$
\begin{gathered}
{\left[\begin{array}{ccc}
U^{*} & 0 & 0 \\
0 & V^{*} & 0 \\
0 & 0 & I_{n-p-q}
\end{array}\right] X\left[\begin{array}{ccc}
U & 0 & 0 \\
0 & V & 0 \\
0 & 0 & I_{n-p-q}
\end{array}\right]} \\
=\left[\begin{array}{ccc}
X_{r} \oplus 0_{p-r} & 0 & 0 \\
0 & -X_{s} \oplus 0_{q-s} & 0 \\
0 & 0 & 0_{n-p-q}
\end{array}\right],
\end{gathered}
$$

where $X_{r} \in H_{r}$ and $X_{s} \in H_{s}$. This, together with (13), implies that $X_{r} \in \mathscr{P}_{r}$ and $X_{s} \in \mathscr{P}_{s}$. Thus,

$$
X=\left[U\left(X_{r} \oplus 0_{p-r}\right) U^{*}\right] \oplus-\left[V\left(X_{s} \oplus 0_{q-s}\right) V^{*}\right] \oplus 0_{n-p-q},
$$

as desired.

Lemma 5. Let $\psi_{1}, \psi_{2}$ be canonical maps on $H_{m_{1} \cdots m_{l}}$, and $U, V$ are invertible matrices of order $m_{1} \cdots m_{l}$. If $\psi_{1}(X) U=V \psi_{2}(X)$ for all $X \in H_{m_{1} \cdots m_{l}}$, then $\psi_{1}=\psi_{2}$ and $U=V=\lambda I_{m_{1} \cdots m_{l}}$ for some $\lambda \neq 0$.

Proof. Let us first point out a simple observation which will be used in our proof. If a matrix $U \in M_{m n}$ commutes with $I_{m} \otimes S$ for all real symmetric $S \in M_{n}$, then $U$ has the form $W \otimes I_{n}$ with $W \in M_{m}$. that

Since $\psi_{1}, \psi_{2}$ are canonical maps on $H_{m_{1} \cdots m_{l}}$, we assume

$$
\psi_{1}=\tau_{1} \otimes \cdots \otimes \tau_{l}, \quad \psi_{2}=\eta_{1} \otimes \cdots \otimes \eta_{l},
$$

where $\tau_{i}$ and $\eta_{i}$ are the identity maps $X \mapsto X$ or the transposition maps $X \mapsto X^{T}$ for $i \in[1, l]$.

Clearly, $\psi_{i}\left(I_{m_{1} \cdots m_{l}}\right)=I_{m_{1} \cdots m_{l}}, i=1,2$, hence $U=V$.

We prove $U=\lambda I_{m_{1} \cdots m_{l}}$ by induction on $l$. The case of $l=1$ is the well-known fact. We assume that the statement holds true for $l-1$. We will prove it for $l$. For any real symmetric $S \in M_{m_{l}}$, since $\psi_{i}\left(I_{m_{1} \cdots m_{l-1}} \otimes S\right)=I_{m_{1} \cdots m_{l-1}} \otimes S, i=1,2$, it follows that $U=W \otimes I_{m_{l}}$ for some matrix $W \in M_{m_{1} \cdots m_{l-1}}$. We define linear maps

$$
\varphi_{1}=\tau_{1} \otimes \cdots \otimes \tau_{l-1}, \quad \varphi_{2}=\eta_{1} \otimes \cdots \otimes \eta_{l-1} .
$$

It is easy to see that $\varphi_{1}$ and $\varphi_{2}$ are canonical maps on $H_{m_{1} \cdots m_{l-1}}$. For any $Y \in H_{m_{1} \cdots m_{l-1}}$, since

$$
\begin{aligned}
\left(\varphi_{1}(Y) W\right) \otimes I_{m_{l}} & =\psi_{1}\left(Y \otimes I_{m_{l}}\right) U \\
& =U \psi_{2}\left(Y \otimes I_{m_{l}}\right) \\
& =\left(W \varphi_{2}(Y)\right) \otimes I_{m_{l}}
\end{aligned}
$$

we have $\varphi_{1}(Y) W=W \varphi_{2}(Y)$, thus, by induction hypothesis, $W=\lambda I_{m_{1} \cdots m_{l-1}}$. Since $U$ is invertible, we have $\lambda \neq 0$. Thus, $U=$ $\lambda I_{m_{1} \cdots m_{l}}$ and $\psi_{1}(X)=(1 / \lambda) \psi_{1}(X) U=(1 / \lambda) U \psi_{2}(X)=\psi_{2}(X)$ for all $X \in H_{m_{1} \cdots m_{l}}$.

Theorem 6. Suppose $\phi$ is a linear map from $H_{m_{1} \cdots m_{l}}$ to $H_{n}$ with $n \leq m_{1} \cdots m_{l}$. Then, $\phi\left(\otimes_{1}^{l} \mathscr{P}_{m_{i}}\right) \subset \mathscr{P}_{n}$ if and only if either $\phi=0$ or $n=m_{1} \cdots m_{l}$, there exists a unitary matrix $U \in M_{n}$ and $a$ canonical map $\pi$ on $H_{m_{1} \cdots m_{l}}$ such that

$$
\phi(X)=U \pi(X) U^{*}, \quad \forall X \in H_{m_{1} \cdots m_{l}} .
$$

Proof. The sufficiency part is obvious. We prove the necessity part by induction on $l$. When $l=1$, it is the result in [13], which has been given in the above introduction. We assume now that the result holds true for $l-1$ and give the proof of the case $l$ by the following five steps.

Step 1. Suppose $P \in \mathscr{P}_{m_{1}}$ and $\phi\left(P \otimes I_{m_{2} \cdots m_{l}}\right)=0_{s} \oplus I_{r} \oplus 0_{n-r-s}$. Then,

$$
\phi(P \otimes X)=0_{s} \oplus \psi(X) \oplus 0_{n-r-s}, \quad \forall X \in H_{m_{2} \cdots m_{l}},
$$

where $\psi$ is a linear map from $H_{m_{2} \cdots m_{l}}$ to $H_{r}$ satisfying $\psi\left(\otimes_{2}^{l} \mathscr{P}_{m_{i}}\right) \subset \mathscr{P}_{r}$.

Proof of Step 1. Set

$$
\begin{gathered}
\Gamma_{0}=\left\{I_{m_{2}} \otimes \cdots \otimes I_{m_{l}}\right\}, \\
\Gamma_{1}=\left\{P_{2} \otimes I_{m_{3} \cdots m_{l}}: P_{2} \in \mathscr{P}_{m_{2}}\right\}, \\
\Gamma_{2}=\left\{P_{2} \otimes P_{3} \otimes I_{m_{4} \cdots m_{l}}: P_{i} \in \mathscr{P}_{m_{i}}, i \in[2,3]\right\}, \\
\cdots \\
\Gamma_{l-1}=\left\{P_{2} \otimes \cdots \otimes P_{m_{l}}: P_{i} \in \mathscr{P}_{m_{i}}, i \in[2, l]\right\} .
\end{gathered}
$$

We prove by induction on $k$ that $\phi(P \otimes X)=0_{s} \oplus \psi(X) \oplus$ $0_{n-r-s}, \forall X \in \Gamma_{k}, k \in[0, l-1]$. Assume for a moment that we have already proved this. Then, when $\phi$ is linear, we can complete the proof of Step 1.

Now, we prove the assertion. The case of $k=0$ is just the assumption. Then, we assume that our statement holds true for $k-1$, and we consider the image of $P \otimes P_{2} \otimes \cdots \otimes P_{k+1} \otimes$ $I_{m_{k+2} \cdots m_{l}}$ for $P_{i} \in \mathscr{P}_{m_{i}}, i \in[2, k+1]$. Because

$$
\begin{aligned}
& P \otimes P_{2} \otimes \cdots \otimes P_{k} \otimes I_{m_{k+1}} \otimes I_{m_{k+2} \cdots m_{l}} \in \otimes_{1}^{l} \mathscr{P}_{m_{i}}, \\
& P \otimes P_{2} \otimes \cdots \otimes P_{k} \otimes P_{k+1} \otimes I_{m_{k+2} \cdots m_{l}} \in \otimes_{1}^{l} \mathscr{P}_{m_{i}}, \\
& P \otimes P_{2} \otimes \cdots \otimes P_{k} \otimes\left(I_{m_{k+1}}-P_{k+1}\right) \otimes I_{m_{k+2} \cdots m_{l}} \in \otimes_{1}^{l} \mathscr{P}_{m_{i}},
\end{aligned}
$$

we obtain using the property of $\phi$ that

$$
\begin{aligned}
& \phi\left(P \otimes P_{2} \otimes \cdots \otimes P_{k} \otimes I_{m_{k+1}} \otimes I_{m_{k+2} \cdots m_{l}}\right) \in \mathscr{P}_{n}, \\
& \phi\left(P \otimes P_{2} \otimes \cdots \otimes P_{k} \otimes P_{k+1} \otimes I_{m_{k+2} \cdots m_{l}}\right) \in \mathscr{P}_{n}, \\
& \phi\left(P \otimes P_{2} \otimes \cdots \otimes P_{k} \otimes I_{m_{k+1}} \otimes I_{m_{k+2} \cdots m_{l}}\right), \\
& \quad-\phi\left(P \otimes P_{2} \otimes \cdots \otimes P_{k+1} \otimes I_{m_{k+2} \cdots m_{l}}\right) \in \mathscr{P}_{n} .
\end{aligned}
$$


We obtain by induction hypothesis and Lemma 2 that $\phi\left(P \otimes P_{2} \otimes \cdots \otimes P_{k+1} \otimes I_{m_{k+2} \cdots m_{l}}\right)=0_{s} \oplus X_{r} \oplus 0_{n-r-s}$, where $X_{r} \in \mathscr{P}_{r}$, as desired.

Step 2. If $m_{1} \cdots m_{l}>n$. Then, $\phi=0$.

Proof of Step 2. Since $E_{i i}^{\left(m_{1}\right)} \otimes I_{m_{2} \cdots m_{l}} \in \otimes_{1}^{l} \mathscr{P}_{m_{i}}, \forall i \in\left[1, m_{1}\right]$ and $\left(E_{i i}^{\left(m_{1}\right)}+E_{j j}^{\left(m_{1}\right)}\right) \otimes I_{m_{2} \cdots m_{l}} \in \otimes_{1}^{l} \mathscr{P}_{m_{i}}, \forall i \neq j \in\left[1, m_{1}\right]$, we obtain using the property of $\phi$ that

$$
\begin{aligned}
& \phi\left(E_{i i}^{\left(m_{1}\right)} \otimes I_{m_{2} \cdots m_{l}}\right) \in \mathscr{P}_{n}, \forall i \in\left[1, m_{1}\right], \\
& \phi\left(E_{i i}^{\left(m_{1}\right)} \otimes I_{m_{2} \cdots m_{l}}\right)+\phi\left(E_{j j}^{\left(m_{1}\right)} \otimes I_{m_{2} \cdots m_{l}}\right) \in \mathscr{P}_{n}, \\
& \forall i \neq j \in\left[1, m_{1}\right] .
\end{aligned}
$$

Denote by $r_{i}=\operatorname{rank} \phi\left(E_{i i}^{\left(m_{1}\right)} \otimes I_{m_{2} \cdots m_{l}}\right), \forall i \in\left[1, m_{1}\right]$. Using Lemma 1 and composing $\phi$ by a similarity transformation, we may obtain that

$$
\phi\left(E_{i i}^{\left(m_{1}\right)} \otimes I_{m_{2} \cdots m_{l}}\right)=\operatorname{diag}(0, \ldots, 0,1, \ldots, 1,0, \ldots, 0),
$$

where $\operatorname{diag}(0, \ldots, 0,1, \ldots, 1,0, \ldots, 0)$ is the diagonal matrix in which all diagonal entries are zero except those in the $\left(r_{1}+\right.$ $\left.\cdots+r_{i-1}+1\right)$ st to the $\left(r_{1}+\cdots+r_{i}\right)$ th rows.

Since $n<m_{1} \cdots m_{l}$, there exists some $r_{i}<m_{2} \cdots m_{l}$. Without loss of generality, we may assume $r_{1}<m_{2} \cdots m_{l}$. Thus, $\phi\left(E_{11}^{\left(m_{1}\right)} \otimes I_{m_{2} \cdots m_{l}}\right)=I_{r_{1}} \oplus 0_{n-r_{1}}$. By Step 1, we see that there exists a linear map $\psi$ from $H_{m_{2} \cdots m_{l}}$ to $H_{r_{1}}$ satisfying $\psi\left(\otimes_{2}^{l} \mathscr{P}_{m_{i}}\right) \subset \mathscr{P}_{r_{1}}$ such that $\phi\left(E_{11}^{\left(m_{1}\right)} \otimes X\right)=\psi(X) \oplus 0_{n-r_{1}}$ for all $X \in H_{m_{2} \cdots m_{l}}$. We obtain by induction hypothesis $\psi=0$. Thus,

$$
\phi\left(E_{11}^{\left(m_{1}\right)} \otimes X\right)=0, \quad \forall X \in H_{m_{2} \cdots m_{l}} .
$$

For any $j \in\left[2, m_{1}\right], P \in \otimes_{2}^{l} \mathscr{P}_{m_{i}}$ and real $\gamma$ with $0 \neq|\gamma| \leq 1 / 2$, we have $\left(\alpha E_{11}^{\left(m_{1}\right)}+\beta E_{j j}^{\left(m_{1}\right)}+\gamma D_{1 j}^{\left(m_{1}\right)}\right) \otimes P \in \otimes_{1}^{l} \mathscr{P}_{m_{i}}$, where $\alpha$ and $\beta$ are all roots of $x^{2}-x+\gamma^{2}=0$. We obtain using the property of $\phi,(27)$, and Lemma 3 that $\phi\left(E_{j j}^{\left(m_{1}\right)} \otimes P\right)=0$ and $\phi\left(D_{1 j}^{\left(m_{1}\right)} \otimes P\right)=0$. By using the similar argument, we see that $\phi\left(D_{i j}^{\left(m_{1}\right)} \otimes P\right)=0, \phi\left(K_{i j}^{\left(m_{1}\right)} \otimes P\right)=0, \forall i \neq j \in\left[1, m_{1}\right]$. Thus,

$$
\phi(A \otimes P)=0, \quad \forall A \in H_{m_{1}} .
$$

By the arbitrariness of $P \in \otimes_{2}^{l} \mathscr{P}_{m_{i}}$, we have

$$
\phi(A \otimes X)=0, \quad \forall A \in H_{m_{1}}, X \in H_{m_{2} \cdots m_{l}} .
$$

Therefore, $\phi=0$.

We next always assume $m_{1} \cdots m_{l}=n$ and $\phi \neq 0$.

Step 3. Suppose $F_{1}, \ldots, F_{m_{1}} \in \mathscr{P}_{m_{1}}$ are of rank one with $F_{i}+$ $F_{j} \in \mathscr{P}_{m_{1}}, \forall i \neq j \in\left[1, m_{1}\right]$. Then, there exists a unitary matrix $U$ of order $m_{1} \cdots m_{l}$ such that

$$
\begin{array}{r}
\phi\left(F_{k} \otimes X\right)=U\left[E_{k k}^{\left(m_{1}\right)} \otimes \pi_{k}(X)\right] U^{*}, \\
\forall X \in H_{m_{2} \cdots m_{l}}, k \in\left[1, m_{1}\right],
\end{array}
$$

where $\pi_{k}$ is a canonical map on $H_{m_{2} \cdots m_{l}}, \forall k \in\left[1, m_{1}\right]$.
Proof of Step 3. Using the similar approach as in the proof of Step 2, we may show that $\operatorname{rank} \phi\left(F_{k} \otimes I_{m_{2} \cdots m_{l}}\right)=m_{2} \cdots m_{l}$, $\forall k \in\left[1, m_{1}\right]$. In fact, if $\operatorname{rank} \phi\left(F_{k} \otimes I_{m_{2} \cdots m_{l}}\right)>m_{2} \cdots m_{l}$ for some $k$, then, by Lemma 1 , there exists $\operatorname{rank} \phi\left(F_{i} \otimes I_{m_{2} \cdots m_{l}}\right)<$ $m_{2} \cdots m_{l}$ for some $i$. Then, using the similar approach as in the proof of Step 2, we have $\phi=0$, which is a contradiction, and there exist a unitary matrix $Q$ such that $\phi\left(F_{k} \otimes I_{m_{2} \cdots m_{l}}\right)=$ $Q\left[E_{k k}^{\left(m_{1}\right)} \otimes I_{m_{2} \cdots m_{l}}\right] Q^{*}$. Thus, applying Step 1, we obtain that

$$
\phi\left(F_{k} \otimes X\right)=Q\left[E_{k k}^{\left(m_{1}\right)} \otimes \psi_{k}(X)\right] Q^{*}, \quad \forall X \in H_{m_{2} \cdots m_{l}},
$$

where linear map $\psi_{k}: H_{m_{2} \cdots m_{l}} \rightarrow H_{m_{2} \cdots m_{l}}$ satisfying $\psi_{k}\left(\otimes_{2}^{l} \mathscr{P}_{m_{i}}\right) \subset \mathscr{P}_{m_{2} \cdots m_{l}}$, for any $k \in\left[1, m_{1}\right]$. We obtain by induction hypothesis that there exists unitary matrices $U_{k} \in$ $\mathscr{M}_{m_{2} \cdots m_{l}}$ and canonical maps $\pi_{k}$ on $H_{m_{2} \cdots m_{l}}$ such that

$$
\psi_{k}(X)=U_{k} \pi_{k}(X) U_{k}^{*}, \quad \forall X \in H_{m_{2} \cdots m_{l}}, k \in\left[1, m_{1}\right] .
$$

Set $U=Q \operatorname{diag}\left(U_{1}, \ldots, U_{m_{1}}\right)$, we complete the proof of Step 3 . By Step 3, we may assume that

$$
\begin{gathered}
\phi\left(E_{k k}^{\left(m_{1}\right)} \otimes X\right)=E_{k k}^{\left(m_{1}\right)} \otimes \pi_{k}(X), \\
\forall X \in H_{m_{2} \cdots m_{l}}, k \in\left[1, m_{1}\right],
\end{gathered}
$$

where $\pi_{k}$ is canonical map on $H_{m_{2} \cdots m_{l}}, \forall k \in\left[1, m_{1}\right]$.

Step 4. For $\forall i \neq j \in\left[1, m_{1}\right], \pi_{i}=\pi_{j}$ and there exists $\lambda_{i j}, \mu_{i j}$ with $\left|\lambda_{i j}\right|=\left|\mu_{i j}\right|=1$ such that

$$
\begin{array}{r}
\phi\left(D_{i j}^{\left(m_{1}\right)} \otimes X\right)=\left(\lambda_{i j} E_{i j}^{\left(m_{1}\right)}+\bar{\lambda}_{i j} E_{j i}^{\left(m_{1}\right)}\right) \otimes \pi_{1}(X), \\
\forall X \in H_{m_{2} \cdots m_{l}}, \\
\phi\left(K_{i j}^{\left(m_{1}\right)} \otimes X\right)=\left(\mu_{i j} E_{i j}^{\left(m_{1}\right)}+\bar{\mu}_{i j} E_{j i}^{\left(m_{1}\right)}\right) \otimes \pi_{1}(X), \\
\forall X \in H_{m_{2} \cdots m_{l}} .
\end{array}
$$

Proof of Step 4. Without loss of generality, we only prove the case of $i=1, j=2$.

Set $F_{1}=(1 / 2) E_{11}^{\left(m_{1}\right)}+(1 / 2) E_{22}^{\left(m_{1}\right)}+(1 / 2) D_{12}^{\left(m_{1}\right)}, F_{2}=$ $(1 / 2) E_{11}^{\left(m_{1}\right)}+(1 / 2) E_{22}^{\left(m_{1}\right)}-(1 / 2) D_{12}^{\left(m_{1}\right)}, F_{k}=E_{k k}^{\left(m_{1}\right)}$ for $k \in\left[3, m_{1}\right]$ (if $m_{1} \geq 3$ ). By Step 3 , there exists a unitary matrix $V$ and canonical maps $\eta_{k}$ on $H_{m_{2} \cdots m_{l}}$ such that

$$
\begin{array}{r}
\phi\left(F_{k} \otimes X\right)=V\left[E_{k k}^{\left(m_{1}\right)} \otimes \eta_{k}(X)\right] V^{*}, \\
\forall X \in H_{m_{2} \cdots m_{l}}, k \in\left[1, m_{1}\right] .
\end{array}
$$

This, together with (33), implies that

$$
E_{k k}^{\left(m_{1}\right)} \otimes I_{m_{2} \cdots m_{l}}=V\left[E_{k k}^{\left(m_{1}\right)} \otimes I_{m_{2} \cdots m_{l}}\right] V^{*}, \quad \forall k \in\left[3, m_{1}\right] .
$$

Thus, we may set

$$
V=\left[\begin{array}{ll}
V_{11} & V_{12} \\
V_{21} & V_{22}
\end{array}\right] \oplus \operatorname{diag}\left(V_{33}, \ldots, V_{m_{1} m_{1}}\right),
$$

where $V_{k k} \in M_{m_{2} \cdots m_{l}}, k \in\left[1, m_{1}\right]$. 
On one hand, noting that

$$
\begin{aligned}
& D_{12}^{\left(m_{1}\right)}=2 F_{1}-E_{11}^{\left(m_{1}\right)}-E_{22}^{\left(m_{1}\right)}, \\
& D_{12}^{\left(m_{1}\right)}=E_{11}^{\left(m_{1}\right)}+E_{22}^{\left(m_{1}\right)}-2 F_{2},
\end{aligned}
$$

we obtain using (33) and (35) that for all $X \in H_{m_{2} \cdots m_{l}}$,

$$
\begin{aligned}
\phi & \left(D_{12}^{\left(m_{1}\right)} \otimes X\right) \\
& =\left[\begin{array}{cc}
2 V_{11} \eta_{1}(X) V_{11}^{*}-\pi_{1}(X) & 2 V_{11} \eta_{1}(X) V_{21}^{*} \\
2 V_{21} \eta_{1}(X) V_{11}^{*} & 2 V_{21} \eta_{1}(X) V_{21}^{*}-\pi_{2}(X)
\end{array}\right] \oplus 0,
\end{aligned}
$$

$$
\begin{aligned}
\phi & \left(D_{12}^{\left(m_{1}\right)} \otimes X\right) \\
& =\left[\begin{array}{cc}
\pi_{1}(X)-2 V_{12} \eta_{2}(X) V_{12}^{*} & -2 V_{12} \eta_{2}(X) V_{22}^{*} \\
-2 V_{22} \eta_{2}(X) V_{12}^{*} & \pi_{2}(X)-2 V_{22} \eta_{2}(X) V_{22}^{*}
\end{array}\right] \oplus 0 .
\end{aligned}
$$

On the other hand, for any $P \in \otimes_{2}^{l} \mathscr{P}_{m_{i}}$ and real $\gamma$ with $0 \neq|\gamma| \leq 1 / 2$, we have $\left(\alpha E_{11}^{\left(m_{1}\right)}+\beta E_{22}^{\left(m_{1}\right)}+\gamma D_{12}^{\left(m_{1}\right)}\right) \otimes P \in \otimes_{1}^{l} \mathscr{P}_{m_{i}}$, where $\alpha$ and $\beta$ are all roots of $x^{2}-x+\gamma^{2}=0$. We obtain using the property of $\phi,(33)$, and Lemma 3 that

$$
\begin{gathered}
{\left[\phi\left(D_{12}^{\left(m_{1}\right)} \otimes I_{m_{2} \cdots m_{l}}\right)\right]^{2}} \\
=\phi\left(E_{11}^{\left(m_{1}\right)} \otimes I_{m_{2} \cdots m_{l}}\right)+\phi\left(E_{22}^{\left(m_{1}\right)} \otimes I_{m_{2} \cdots m_{l}}\right), \\
\phi\left(D_{12}^{\left(m_{1}\right)} \otimes X\right)=\left[\begin{array}{ll}
0_{m_{1}} & Y_{12} \\
Y_{12}^{*} & 0_{m_{1}}
\end{array}\right] \oplus 0, \quad \forall X \in H_{m_{2} \cdots m_{l}},
\end{gathered}
$$

where $Y_{12} \in M_{m_{1}}$. Choosing $X=I_{m_{2} \cdots m_{l}}$, we get using (39), (40), and (42) that $2 V_{11} V_{11}^{*}=2 V_{22} V_{22}^{*}=2 V_{21} V_{21}^{*}=2 V_{12} V_{12}^{*}=$ $I_{m_{2} \cdots m_{l}}$. Hence, by (39), (40), and (42) again, one can obtain that

$$
\begin{aligned}
& \pi_{1}(X)\left(V_{11}^{*}\right)^{-1} V_{21}^{*} \\
& \quad=2 V_{11} \eta_{1}(X) V_{21}^{*}=-2 V_{12} \eta_{2}(X) V_{22}^{*}=-V_{12} V_{22}^{-1} \pi_{2}(X) .
\end{aligned}
$$

By Lemma 5, we have $\pi_{1}=\pi_{2}$ and $\left(V_{11}^{*}\right)^{-1} V_{21}^{*}=\lambda_{12} I_{m_{2} \cdots m_{l}}$ with $\lambda_{12} \neq 0$. Hence,

$$
\begin{array}{r}
\phi\left(D_{12}^{\left(m_{1}\right)} \otimes X\right)=\left(\lambda_{12} E_{12}^{\left(m_{1}\right)}+\bar{\lambda}_{12} E_{21}^{\left(m_{1}\right)}\right) \otimes \pi_{1}(X), \\
\forall X \in H_{m_{2} \cdots m_{l}}, \lambda_{12} \neq 0 .
\end{array}
$$

This, together with (41), implies that $\left|\lambda_{12}\right|=1$. Similarly,

$$
\begin{array}{r}
\phi\left(K_{12}^{\left(m_{1}\right)} \otimes X\right)=\left(\mu_{12} E_{12}^{\left(m_{1}\right)}+\bar{\mu}_{12} E_{21}^{\left(m_{1}\right)}\right) \otimes \pi_{1}(X), \\
\forall X \in H_{m_{2} \cdots m_{l}},\left|\mu_{12}\right|=1 .
\end{array}
$$

This completes the proof of Step 4.
Step 5. There exists $\varepsilon \in\{-1,1\}$ such that $\mu_{i j}=\varepsilon \sqrt{-1} \lambda_{i j}$, $\forall i \neq j \in\left[1, m_{1}\right]$. If $m_{1} \geq 3$, then $\lambda_{i j} \lambda_{j k}=\lambda_{i k}$, for distinct $i, j, k \in\left[1, m_{1}\right]$.

Proof of Step 5. Since

$$
\begin{gathered}
\left(\frac{1}{2} E_{i i}^{\left(m_{1}\right)}+\frac{1}{2} E_{j j}^{\left(m_{1}\right)}+\frac{\sqrt{2}}{4} D_{i j}^{\left(m_{1}\right)}+\frac{\sqrt{2}}{4} K_{i j}^{\left(m_{1}\right)}\right) \\
\otimes I_{m_{2} \cdots m_{l}} \in \otimes_{1}^{l} \mathscr{P}_{m_{i}},
\end{gathered}
$$

we obtain using the property of $\phi,(33)$, and Step 4 that

$$
\left[\begin{array}{cc}
\frac{1}{2} & \frac{\sqrt{2}}{4}\left(\lambda_{i j}+\mu_{i j}\right) \\
\frac{\sqrt{2}}{4}\left(\bar{\lambda}_{i j}+\bar{\mu}_{i j}\right) & \frac{1}{2}
\end{array}\right] \in \mathscr{P}_{2} .
$$

Hence, $\left(\lambda_{i j}+\mu_{i j}\right)\left(\bar{\lambda}_{i j}+\bar{\mu}_{i j}\right)=2$. It follows from $\left|\lambda_{i j}\right|=\left|\mu_{i j}\right|=1$ that $\lambda_{i j}^{2}=-\mu_{i j}^{2}$. Thus, there exists $\varepsilon_{i j} \in\{-1,1\}$ such that $\mu_{i j}=$ $\varepsilon_{i j} \sqrt{-1} \lambda_{i j}$.

If $m_{1} \geq 3$, since

$$
\begin{aligned}
& \frac{1}{3}\left(E_{i i}^{\left(m_{1}\right)}+E_{j j}^{\left(m_{1}\right)}+E_{k k}^{\left(m_{1}\right)}+D_{i j}^{\left(m_{1}\right)}+D_{i k}^{\left(m_{1}\right)}+D_{j k}^{\left(m_{1}\right)}\right) \\
& \otimes I_{m_{2} \cdots m_{l}} \in \otimes_{1}^{l} \mathscr{P}_{m_{i}}, \\
& \frac{1}{3}\left(E_{i i}^{\left(m_{1}\right)}+E_{j j}^{\left(m_{1}\right)}+E_{k k}^{\left(m_{1}\right)}+K_{i j}^{\left(m_{1}\right)}+K_{i k}^{\left(m_{1}\right)}+D_{j k}^{\left(m_{1}\right)}\right) \\
& \otimes I_{m_{2} \cdots m_{l}} \in \otimes_{1}^{l} \mathscr{P}_{m_{i}}, \\
& \frac{1}{3}\left(E_{i i}^{\left(m_{1}\right)}+E_{j j}^{\left(m_{1}\right)}+E_{k k}^{\left(m_{1}\right)}+D_{i j}^{\left(m_{1}\right)}+K_{i k}^{\left(m_{1}\right)}+K_{j k}^{\left(m_{1}\right)}\right) \\
& \otimes I_{m_{2} \cdots m_{l}} \in \otimes_{1}^{l} \mathscr{P}_{m_{i}},
\end{aligned}
$$

we obtain using the property of $\phi,(33)$, and Step 4 that

$$
\begin{array}{r}
\frac{1}{3}\left[\begin{array}{ccc}
1 & \lambda_{i j} & \lambda_{i k} \\
\bar{\lambda}_{i j} & 1 & \lambda_{j k} \\
\bar{\lambda}_{i k} & \bar{\lambda}_{j k} & 1
\end{array}\right] \in \mathscr{P}_{3}, \\
\frac{1}{3}\left[\begin{array}{ccc}
1 & \varepsilon_{i j} \lambda_{i j} \sqrt{-1} & \varepsilon_{i k} \lambda_{i k} \sqrt{-1} \\
-\varepsilon_{i j} \bar{\lambda}_{i j} \sqrt{-1} & 1 & \lambda_{j k} \\
-\varepsilon_{i k} \bar{\lambda}_{i k} \sqrt{-1} & \bar{\lambda}_{j k} & 1
\end{array}\right] \in \mathscr{P}_{3}, \\
\frac{1}{3}\left[\begin{array}{ccc}
1 & \lambda_{i j} & \varepsilon_{i k} \lambda_{i k} \sqrt{-1} \\
\bar{\lambda}_{i j} & 1 & \varepsilon_{j k} \lambda_{j k} \sqrt{-1} \\
-\varepsilon_{i k} \bar{\lambda}_{i k} \sqrt{-1} & -\varepsilon_{j k} \bar{\lambda}_{j k} \sqrt{-1} & 1
\end{array}\right] \in \mathscr{P}_{3} .
\end{array}
$$

It follows from (49) that $\lambda_{i j} \lambda_{j k}=\lambda_{i k}$. This, together with (50), implies that $\varepsilon_{i j}=\varepsilon_{i k}$. Similarly, (51) implies that $\varepsilon_{i k}=\varepsilon_{j k}$. Thus, all numbers $\varepsilon_{i j}$ have to be the same for any $i \neq j \in$ $\left[1, m_{1}\right]$. This completes the proof of this step. 
By Steps 3-5, we set $Q=\operatorname{diag}\left(1, \lambda_{12}, \ldots, \lambda_{1 m_{1}}\right)$, then $Q$ is unitary such that

$$
\begin{array}{r}
\phi\left(E_{k k}^{\left(m_{1}\right)} \otimes X\right)=\left[Q E_{k k}^{\left(m_{1}\right)} Q^{*}\right] \otimes \pi_{1}(X), \\
\forall X \in H_{m_{2} \cdots m_{l}}, k \in\left[1, m_{1}\right], \\
\phi\left(D_{i j}^{\left(m_{1}\right)} \otimes X\right)=\left[Q D_{i j}^{\left(m_{1}\right)} Q^{*}\right] \otimes \pi_{1}(X), \\
\forall X \in H_{m_{2} \cdots m_{l}}, i \neq j \in\left[1, m_{1}\right], \\
\phi\left(K_{i j}^{\left(m_{1}\right)} \otimes X\right)=\varepsilon\left[Q K_{i j}^{\left(m_{1}\right)} Q^{*}\right] \otimes \pi_{1}(X), \\
\forall X \in H_{m_{2} \cdots m_{l}}, i \neq j \in\left[1, m_{1}\right] .
\end{array}
$$

Thus, set $U=Q \otimes I_{m_{2} \cdots m_{l}}$, we have

$$
\begin{array}{r}
\phi(A \otimes X)=U\left[A \otimes \pi_{1}(X)\right] U^{*}, \\
\forall A \in H_{m_{1}}, \quad X \in H_{m_{2} \cdots m_{l}}
\end{array}
$$

or

$$
\begin{array}{r}
\phi(A \otimes X)=U\left[A^{T} \otimes \pi_{1}(X)\right] U^{*}, \\
\forall A \in H_{m_{1}}, X \in H_{m_{2} \cdots m_{l}} .
\end{array}
$$

This completes the proof of Theorem 6 .

Remark 7. When $l \geq 2$, the linear transformation that maps the set of tensor product of idempotent matrices into idempotent matrix set does not necessarily preserve idempotent matrices. For example, let

$$
\begin{aligned}
& \phi\left(A_{1} \otimes A_{2} \otimes \cdots \otimes A_{l}\right)=A_{1}^{T} \otimes A_{2} \otimes \cdots \otimes A_{l}, \\
& \forall A_{i} \in \mathscr{H}_{m_{i}}, i \in[1, l], \\
& C=\frac{1}{2}\left(E_{11}^{\left(m_{1}\right)} \otimes E_{11}^{\left(m_{2}\right)}+E_{22}^{\left(m_{1}\right)} \otimes E_{22}^{\left(m_{2}\right)}+E_{12}^{\left(m_{1}\right)}\right. \\
&\left.\otimes E_{12}^{\left(m_{2}\right)}+E_{21}^{\left(m_{1}\right)} \otimes E_{21}^{\left(m_{2}\right)}\right) \otimes I_{m_{3} \cdots m_{l}} .
\end{aligned}
$$

Then $\phi\left(\otimes_{1}^{l} \mathscr{P}_{m_{i}}\right) \subset \mathscr{P}_{m_{1} \cdots m_{l}}$ and $C \in \mathscr{P}_{m_{1} \cdots m_{l}}$, but

$$
\begin{aligned}
& \phi(C)=\frac{1}{2}( E_{11}^{\left(m_{1}\right)} \otimes E_{11}^{\left(m_{2}\right)}+E_{22}^{\left(m_{1}\right)} \otimes E_{22}^{\left(m_{2}\right)}+E_{21}^{\left(m_{1}\right)} \\
&\left.\otimes E_{12}^{\left(m_{2}\right)}+E_{12}^{\left(m_{1}\right)} \otimes E_{21}^{\left(m_{2}\right)}\right) \otimes I_{m_{3} \cdots m_{l}} \notin \mathscr{P}_{m_{1} \cdots m_{l}} .
\end{aligned}
$$

As the application of Theorem 6, we give the following theorem.

Theorem 8. Suppose $\phi$ is a linear map from $H_{m_{1} \cdots m_{l}}$ to $H_{n}$ with $n \leq m_{1} \cdots m_{l}$. Then, $\phi\left(\otimes_{1}^{l} \mathscr{T}_{m_{i}}\right) \subset \mathscr{T}_{n}$ if and only if either $\phi=0$ or $n=m_{1} \cdots m_{l}$, there exist a unitary matrix $U \in M_{n}, \varepsilon \in$ $\{-1,1\}$ and a canonical map $\pi$ on $H_{m_{1} \cdots m_{l}}$ such that

$$
\phi(X)=\varepsilon U \pi(X) U^{*}, \quad \forall X \in H_{m_{1} \cdots m_{l}} .
$$

Proof. The sufficiency part is clear. We give the proof of the necessity part.

Set

$$
\begin{gathered}
\Gamma_{0}=\left\{I_{m_{1}} \otimes \cdots \otimes I_{m_{l}}\right\}, \\
\Gamma_{1}=\left\{P_{1} \otimes I_{m_{2} \cdots m_{l}}: P_{1} \in \mathscr{P}_{m_{1}}\right\}, \\
\Gamma_{2}=\left\{P_{1} \otimes P_{2} \otimes I_{m_{3} \cdots m_{l}}: P_{i} \in \mathscr{P}_{m_{i}}, i \in[1,2]\right\}, \\
\cdots \\
\Gamma_{l}=\left\{P_{1} \otimes \cdots \otimes P_{m_{l}}: P_{i} \in \mathscr{P}_{m_{i}}, i \in[1, l]\right\} .
\end{gathered}
$$

It is obvious that $\Gamma_{l}=\otimes_{1}^{l} \mathscr{P}_{m_{i}}$ and $\Gamma_{k} \subset \otimes_{1}^{l} \mathscr{T}_{m_{i}}, \forall k \in[0, l]$.

Since $I_{m_{1} \cdots m_{l}} \in \otimes_{1}^{l} \mathscr{T}_{m_{i}}$, we have $\phi\left(I_{m_{1} \cdots m_{l}}\right) \in \mathscr{T}_{n}$. Without loss of generality, we may assume that $\phi\left(I_{m_{1} \cdots m_{l}}\right)=I_{p} \oplus-I_{q} \oplus$ $0_{n-p-q}$. We next prove by induction on $k$ that

$$
\phi(X)=\psi_{p}(X) \oplus-\psi_{q}(X) \oplus 0_{n-p-q}, \quad \forall X \in \Gamma_{k},
$$

where $\psi_{p}(X) \in \mathscr{P}_{p}$ and $\psi_{q}(X) \in \mathscr{P}_{q}$.

We assume that our statement holds true for $k-1$ and prove it for $k$. For any $X=P_{1} \otimes \cdots \otimes P_{k} \otimes I_{m_{k+1} \cdots m_{l}} \in \Gamma_{k}$, choosing $Y=P_{1} \otimes \cdots \otimes P_{k-1} \otimes I_{m_{k}} \otimes I_{m_{k+1} \cdots m_{l}} \in \Gamma_{k-1}$, we have by induction hypothesis that

$$
\phi(Y)=\psi_{p}(Y) \oplus-\psi_{q}(Y) \oplus 0_{n-p-q},
$$

with $\psi_{p}(Y) \in \mathscr{P}_{p}$ and $\psi_{q}(Y) \in \mathscr{P}_{q}$. Since $X \in \otimes_{1}^{l} \mathscr{T}_{m_{i}}, Y-X \in$ $\otimes_{1}^{l} \mathscr{T}_{m_{i}}$ and $Y-2 X \in \otimes_{1}^{l} \mathscr{T}_{m_{i}}$. Using the property of $\phi$, we have $\phi(X) \in \mathscr{T}_{n}, \phi(Y)-\phi(X) \in \mathscr{T}_{n}$ and $\phi(Y)-2 \phi(X) \in \mathscr{T}_{n}$. By Lemma 4 , we have

$$
\phi(X)=\psi_{p}(X) \oplus-\psi_{q}(X) \oplus 0_{n-p-q},
$$

with $\psi_{p}(X) \in \mathscr{P}_{p}$ and $\psi_{q}(X) \in \mathscr{P}_{q}$. This implies that (59) holds.

As $\phi$ is linear, we can expend $\psi_{p}$ to be a linear map from $H_{m_{1} \cdots m_{l}}$ to $H_{p}$ and $\psi_{q}$ to be a linear map from $H_{m_{1} \cdots m_{l}}$ to $H_{q}$, then

$$
\phi(X)=\psi_{p}(X) \oplus-\psi_{q}(X) \oplus 0_{n-p-q}, \quad \forall X \in H_{m_{1} \cdots m_{l}},
$$

with $\psi_{p}\left(\otimes_{1}^{l} \mathscr{P}_{m_{i}}\right) \subset \mathscr{P}_{p}$ and $\psi_{q}\left(\otimes_{1}^{l} \mathscr{P}_{m_{i}}\right) \subset \mathscr{P}_{q}$, we obtain using Theorem 6 that

(a) if $n<m_{1} \cdots m_{l}$, then $p, q<m_{1} \cdots m_{l}, \psi_{p}=0, \psi_{q}=0$, thus, $\phi=0$, and

(b) if $\phi \neq 0$, then $n=m_{1} \cdots m_{l}$ and $p=n$ or $q=n$, thus $\phi=\psi_{p}$ or $\phi=-\psi_{q}$.

This completes the proof of Theorem 8 .

\section{Acknowledgments}

The authors show great thanks to the referee for his/her careful reading of the paper and valuable comments which greatly improved the readability of the paper. Jinli $\mathrm{Xu}$ is supported 
in part by NSFC (11171294), Natural Science Foundation of Heilongjiang Province of China (Grant no. A201013). Baodong Zheng is supported by the National Natural Science Foundation Grants of China (Grant no. 10871056). Hongmei Yao is supported by the Fundamental Research Funds for the Central Universities.

\section{References}

[1] A. Fošner, Z. Haung, C. K. Li, and N. S. Sze, "Linear preservers and quantum information science," Linear and Multilinear Algebra, 2012.

[2] K. He, J. C. Hou, and C. K. Li, "A geometric characterization of invertible quantum measurement maps," Journal of Functional Analysis, vol. 264, no. 2, pp. 464-478, 2013.

[3] S. Friedland, C. K. Li, Y. T. Poon, and N. S. Sze, “The automorphism group of separable states in quantum information theory," Journal of Mathematical Physics, vol. 52, no. 4, Article ID 042203, 8 pages, 2011.

[4] M. A. Nielsen and I. L. Chuang, Quantum Computation and Quantum Information, Cambridge University Press, Cambridge, UK, 2000.

[5] M. H. Lim, "Additive preservers of tensor product of rank one Hermitian matrices," Electronic Journal of Linear Algebra, vol. 23, pp. 356-374, 2012.

[6] Z. H. Zhou and M. Zhu, "Extended Cesáro operators between generalized Besov spaces and Bloch type spaces in the unit ball," Journal of Function Spaces and Applications, vol. 7, no. 3, pp. 209-223, 2009.

[7] H. Yao and B. Zheng, "Zero triple product determined matrix algebras," Journal of Applied Mathematics, vol. 2012, Article ID 925092, 18 pages, 2012.

[8] A. El-Sayed Ahmed and M. A. Bakhit, "Properties of Toeplitz operators on some holomorphic Banach function spaces," Journal of Function Spaces and Applications, vol. 2012, Article ID 517689, 18 pages, 2012.

[9] Z. Liao, S. Hu, D. Sun, and W. Chen, "Enclosed Laplacian operator of nonlinear anisotropic diffusion to preserve singularities and delete isolated points in image smoothing," Mathematical Problems in Engineering, vol. 2011, Article ID 749456, 15 pages, 2011.

[10] O. Agratini, "Linear operators that preserve some test functions," International Journal of Mathematics and Mathematical Sciences, vol. 2006, Article ID 94136, 11 pages, 2006.

[11] D. Huo, B. Zheng, and H. Liu, "Characterizations of nonlinear Lie derivations of $B(X)$," Abstract and Applied Analysis, vol. 2013, Article ID 245452, 7 pages, 2013.

[12] G. H. Chan, M. H. Lim, and K. K. Tan, "Linear preservers on matrices," Linear Algebra and Its Applications, vol. 93, pp. 6780, 1987.

[13] C. H. Sheng, C. G. Cao, and J. Hu, "Linear preservers on complex Hermitian matrices," Journal of Natural Science of Heilongjiang University, vol. 25, pp. 358-362, 2008 (Chinese).

[14] L. Molnár, "Transformations on the set of all $n$-dimensional subspaces of a Hilbert space preserving principal angles," Communications in Mathematical Physics, vol. 217, no. 2, pp. 409-421, 2001.

[15] L. Molnár, "Orthogonality preserving transformations on indefinite inner product spaces: generalization of Uhlhorn's version of Wigner's theorem," Journal of Functional Analysis, vol. 194, no. 2, pp. 248-262, 2002.
[16] P. Šemrl, "Applying projective geometry to transformations on rank one idempotents," Journal of Functional Analysis, vol. 210, no. 1, pp. 248-257, 2004.

[17] P. Šemrl, "Maps on idempotent matrices over division rings," Journal of Algebra, vol. 298, no. 1, pp. 142-187, 2006.

[18] N. Jacobson, Lectures in Abstract Algebra, vol. 2, Van Nostrand, Toronto, Canada, 1953. 


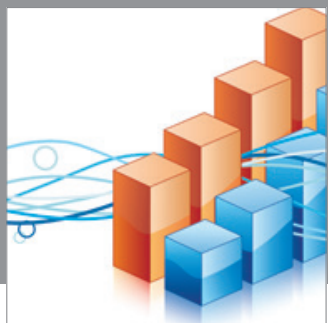

Advances in

Operations Research

mansans

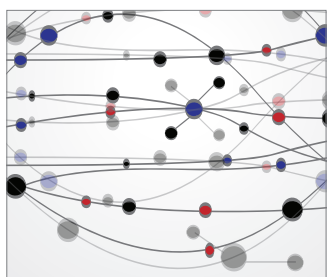

The Scientific World Journal
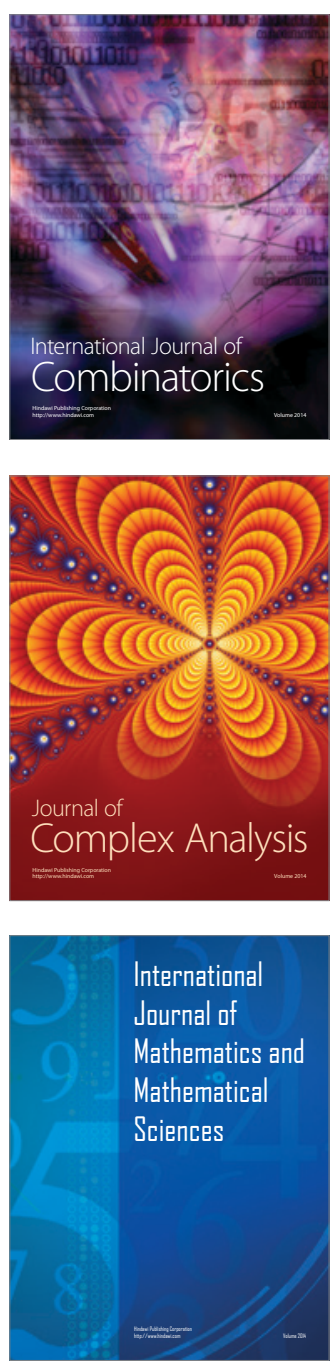
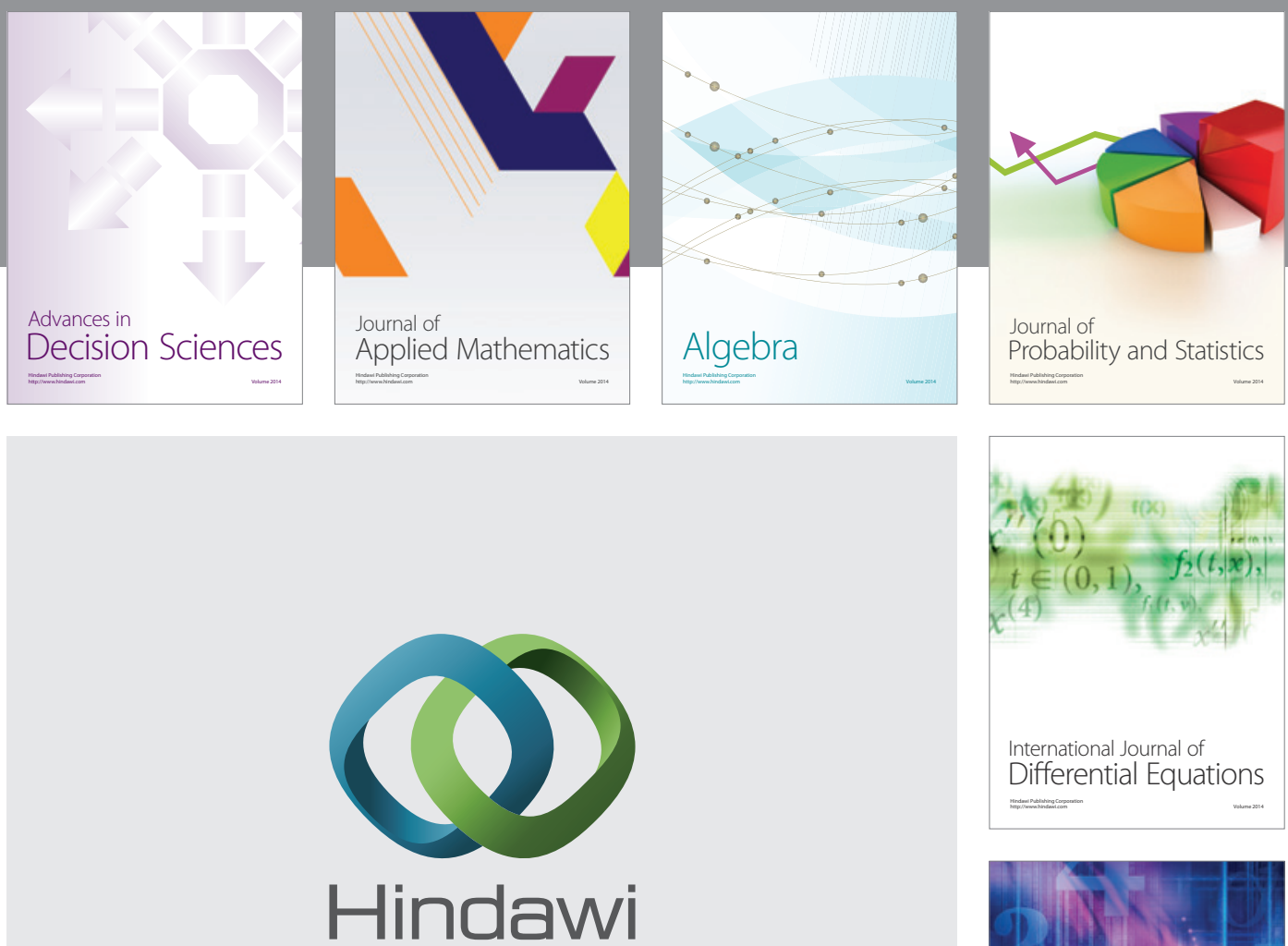

Submit your manuscripts at http://www.hindawi.com
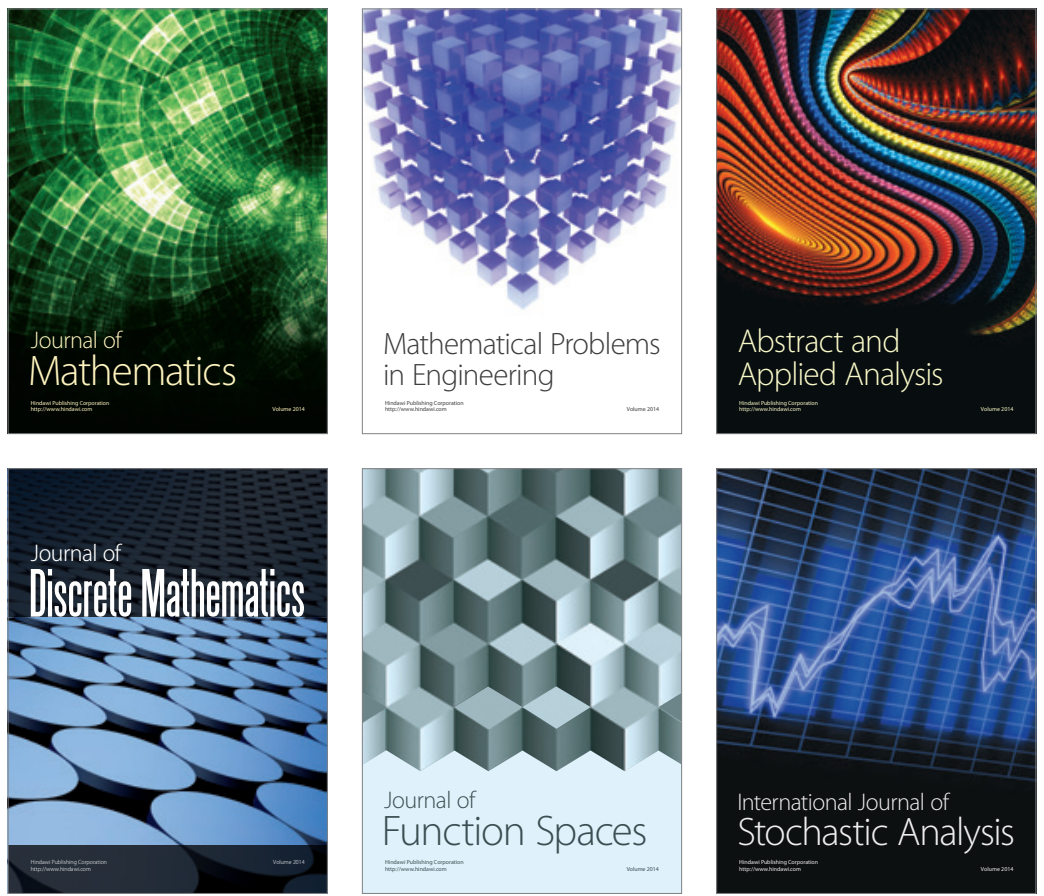

Journal of

Function Spaces

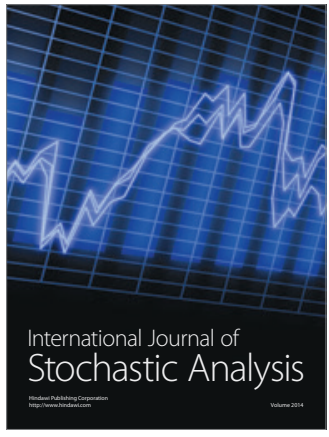

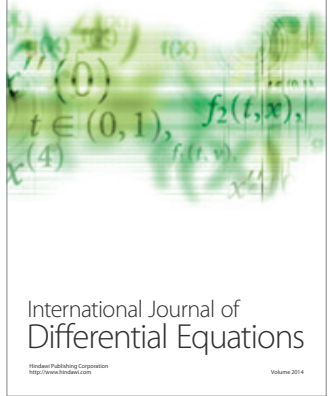
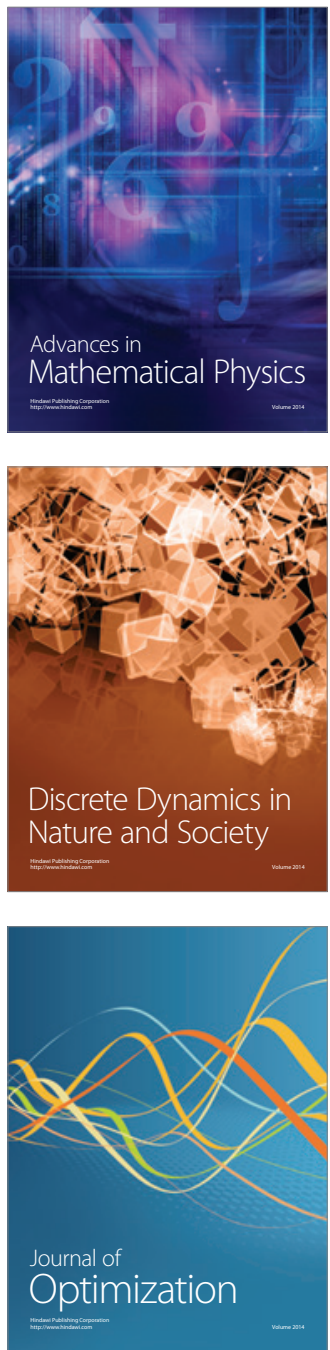